\title{
ESPACIOS ECOLÓGICO-CULTURALES EN UN TERRITORIO MAPUCHE DE LA REGIÓN DE LA ARAUCANÍA EN CHILE
}

\author{
ECOLOGIC-CULTURAL SPACES OF A MAPUCHE TERRITORY \\ IN THE ARAUCANIA REGION-CHILE
}

\author{
Zoia Neira Ceballos ${ }^{1}$, Ana M. Alarcón ${ }^{2 *}$, Ivonne Jelves ${ }^{3}$, Paz Ovalle ${ }^{1}$, \\ Ana M. Conejeros ${ }^{4}$ y Vanessa Verdugo ${ }^{1}$
}

\begin{abstract}
Estudio cualitativo, basado en la metodología de investigación acción participativa, que examina las categorías y significados que los Mapuche de Boroa-Filu-Lawen en Chile poseen de los espacios ecológicos de su territorio. Sus objetivos son identificar y caracterizar los espacios ecológicos Mapuche y determinar su importancia cultural o religiosa. La información la proporcionan 14 habitantes Mapuche, a través de entrevistas grupales e individuales, y visitas de reconocimiento en terreno. Los resultados señalan la presencia de 14 espacios ecológico-culturales, significativos por su interrelación entre elementos culturales, vegetación predominante, relación con el agua y ubicación topográfica. La concepción de espacio trasciende a sus características ecológicas; implica la presencia de seres y fuerzas espirituales que le otorgan su sentido cultural y le confieren poderes espirituales o medicinales. Desde una perspectiva biofísica, los espacios descritos de mayor frecuencia e importancia son los relacionados con el agua, porque proporciona la humedad suficiente para el desarrollo de especies vegetales culturalmente significativas, en su uso medicinal o ceremonial. El conocimiento tradicional Mapuche sobre los espacios ecológicos es fundamental tanto para la preservación de la cultura propia como para diseñar programas de conservación territorialmente pertinentes.
\end{abstract}

Palabras claves: espacios ecológico-culturales, sitios de valor cultural, etnobotánica, conocimiento indígena, Mapuche.

This is a qualitative study based on a participative action investigation that examines categories and meanings of Mapuche ecological and cultural spaces, in Boroa-Filulawen, Chile. The objectives are identification of ecological spaces and their cultural importance. Data were collected during 14 in depth interviews with Mapuche informants during field visits. The results indicate 14 cultural-ecological spaces of special meaning to the Mapuche. These spaces were valued because of their close relationship with Mapuche cultural cosmogony, landscape, vegetation, rivers and other water sources and their location. For the Mapuche, landscape means more than simply geographical and ecological characteristics; it involves spiritual forces, personifications of elements and mystical entities and beings within the landscape. From a biophysical perspective, the spaces more frequently described are those having adequate water supplies for the growth of vegetable species. These plants may supply foods, medicines, clothing, or be used for special religious or ceremonial occasions. This traditional Mapuche knowledge of ecological spaces is important to preserve the culture as well as to design conservation programs territorially pertinent.

Key words: Ecologic-cultural spaces, ethnobotany, indigenous knowledge, Mapuche.

En Chile, las economías locales rurales comienzan a dar cuenta de los efectos de la sustitución de extensas zonas de bosques y otros ecosistemas nativos por monocultivos, tanto de especies forestales exóticas, como de especies agrícolas. Fenómeno al que se agregan la experimentación biogenética para aumentar rendimientos y control de plagas (Altieri 2003; Hill 2006; Marvier 2002; Pérez 2004).

Este modelo de plantaciones genera una serie de impactos negativos como: deforestación, agotamiento de recursos hídricos, erosión del suelo, y desaparición de las plantas y animales locales (Mackinnon y McFall 2000). La pérdida creciente del bosque nativo ha significado un daño a la biodiversidad de la cual se nutre y mantiene el equilibrio de un ecosistema; estos cambios no sólo influyen en la subsistencia de las comunidades indígenas, sino también en la relación hombre-naturaleza, y en la transmisión del conocimiento ancestral sobre los ecosistemas (Lara 1987; La Torre 2003; Toledo 1990). Diversos pueblos originarios y comunidades campesinas han basado su subsistencia en el

1 Departamento de Ciencias Forestales, Facultad de Ciencias Agropecuarias y Forestales, Universidad de La Frontera, Temuco, Chile, Francisco Salazar 01145, Temuco, Chile. Fono- Fax: 56-45-325641. zneira@ufro.cl

2 Departamento de Salud Pública-CIGES, Facultad de Medicina, Universidad de La Frontera, Temuco, Chile. Universidad de La Frontera, Chile. *autor correspondiente: amalarc@ufro.cl

3 Seremia de Salud, Región de Los Ríos, Chile, Valdivia, Chile. ivonne.jelves@ redsalud.gov.cl

4 Departamento Salud Municipalidad de Imperial, Imperial, Chile. aconejero@ hotmail.com 
principio de sostenibilidad, basado en una relación de equilibrio con la naturaleza (Kristensen y Balslev 2003). Los mapuche, pueblo originario de Chile, mantienen una relación de reciprocidad con todos los elementos que conforman la naturaleza, ya sean aves, animales, insectos, plantas, piedras, aguas, e incluso seres espirituales que habitan estos espacios, esto ha sido ampliamente documentado desde los primeros cronistas que llegaron a Chile hasta investigadores contemporáneos (Citarella et al. 2000; Gusinde 1936, 1938; Pérez 2005). Esta relación se sustenta en un modelo filosófico sobre el origen del pueblo mapuche, en que una misma energía o Newen crea al Chen (hombre-mujer) y al Mapu (lugar donde habita el chen), por ello todos están conectados espiritual y materialmente, constituyendo parte de un mismo origen (Marileo 2000). Como una forma de explicarse el universo o cosmos, los mapuche han identificado una serie de espacios cósmico-culturales que dan sentido a la convivencia del hombre-mujer con la naturaleza, los ancestros o antepasados, y los espíritus que pueblan estos espacios (Gusinde 1936; Grebe 1972). Marileo (1995) plantea además la existencia de un espacio simbólico, cultural y material llamado Wallme, el cual representa un micromundo de alto valor y responsabilidad para el sujeto. Se simboliza como un círculo que alcanza todo aquello que se puede observar y contiene todo lo que en él habita; según el autor este espacio debe cuidarse y resguardarse como mapuche; asimismo Nanculef (1989) nomina a este espacio Wallontu Mapu que significa "círculo, o todo lo que nos rodea". Este ordenamiento natural y espiritual se ha traducido en una identificación, nominación y significación de los hábitats o unidades ecológicas mapuche, en los que claramente se diferencian espacios rituales o sagrados, de sustento familiar, de crecimiento de especies medicinales, de realización de la vida cotidiana, etc. (Bragg et al. 1986; Marileo 2001; Villagrán et al. 2003). A su vez Gumucio (1989) plantea que en la nominación y uso del mundo vegetal se observa articulación entre lo cultural, filosófico y cósmico, lo que indicaría que el sistema botánico mapuche es "participativo, holístico y totalizante", haciendo alusión a que en el uso de las plantas es posible observar estos vínculos sociales, materiales y espirituales.

Este trabajo, realizado en conjunto con dirigentes de una comunidad mapuche, explora la presencia de espacios ecológicos en un territorio determinado según sus concepciones culturales. El estudio se desarrolló en el territorio de BoroaFilulawen, ubicado en la Comuna de Nueva Imperial, Región de La Araucanía, Chile. La Figura 1 muestra el territorio de estudio, el cual tiene una superficie de 4.729 has, allí habitan 1.560 personas agrupadas en 380 familias provenientes de 16 comunidades del sector (Comité de Salud Intercultural Boroa-Filulawen 2002). Nueva Imperial posee una población de 40.059 habitantes de los cuales un 53,4\% corresponde a población mapuche (INE 2002).

El clima de la zona corresponde al tipo templado Mesotermal Inferior Estenotérmico Mediterráneo Húmedo de Serranías costeras de vertiente oriental. El régimen térmico varía en promedio, entre una máxima de $24,1{ }^{\circ} \mathrm{C}$ en enero y una mínima de $4,1^{\circ} \mathrm{C}$ en julio. El régimen hídrico presenta una precipitación media anual de $1.342 \mathrm{~mm}$ (Santibáñez y Uribe 1993).

El territorio se ubica en la depresión intermedia de la región, caracterizado por lomajes suaves y con pendientes complejas, lo cual determina la susceptibilidad de erosión de estos suelos que se clasifican del tipo rojo arcilloso, serie Metrenco, de origen volcánico antiguo (Municipalidad de Nueva Imperial 2004). La comuna tiene 10.000 ha erosionadas y $66 \%$ de riesgo de erosión de sus suelos, ocasionado por uso agrícola inadecuado, siembra en suelos no arables, sobrepastoreo, y forestación exótica, entre otras (Antivil 2004, CIREN 2010).

Nueva Imperial corresponde al bosque caducifolio de La Frontera, conformado por Nothofagus obliqua-Nothofagus dombeyi (Roble-Coigüe), Drimys winteri-Blepharocalyx divaricatum (Canelo-Temu), Avena fatua-Rumex acetosella (Teatina-Vinagrillo), Aristotelia chilensis-Rubis ulmifolius (Maqui-Murra), Echium vulgare (Hierba Azul) y Acacia dealbata (Aromo) (Gajardo 1994).

Los cultivos típicos de la zona son: trigo, avena, cebada y papa (ODEPA 2000), y un $2 \%$ de la superficie de la comuna está cubierta con bosque, principalmente con plantaciones exóticas (CONAF y CONAMA 1999). Los mapuche de Boroa-Filulawen dan cuenta de la pérdida cultural que se ha producido en el territorio, debido a la presión aculturadora ejercida desde el Estado chileno. Refieren pérdida de la lengua, credibilidad en las machi, conocimiento y costumbres ancestrales, entre otras. Por tratar de generar recursos a corto plazo, 


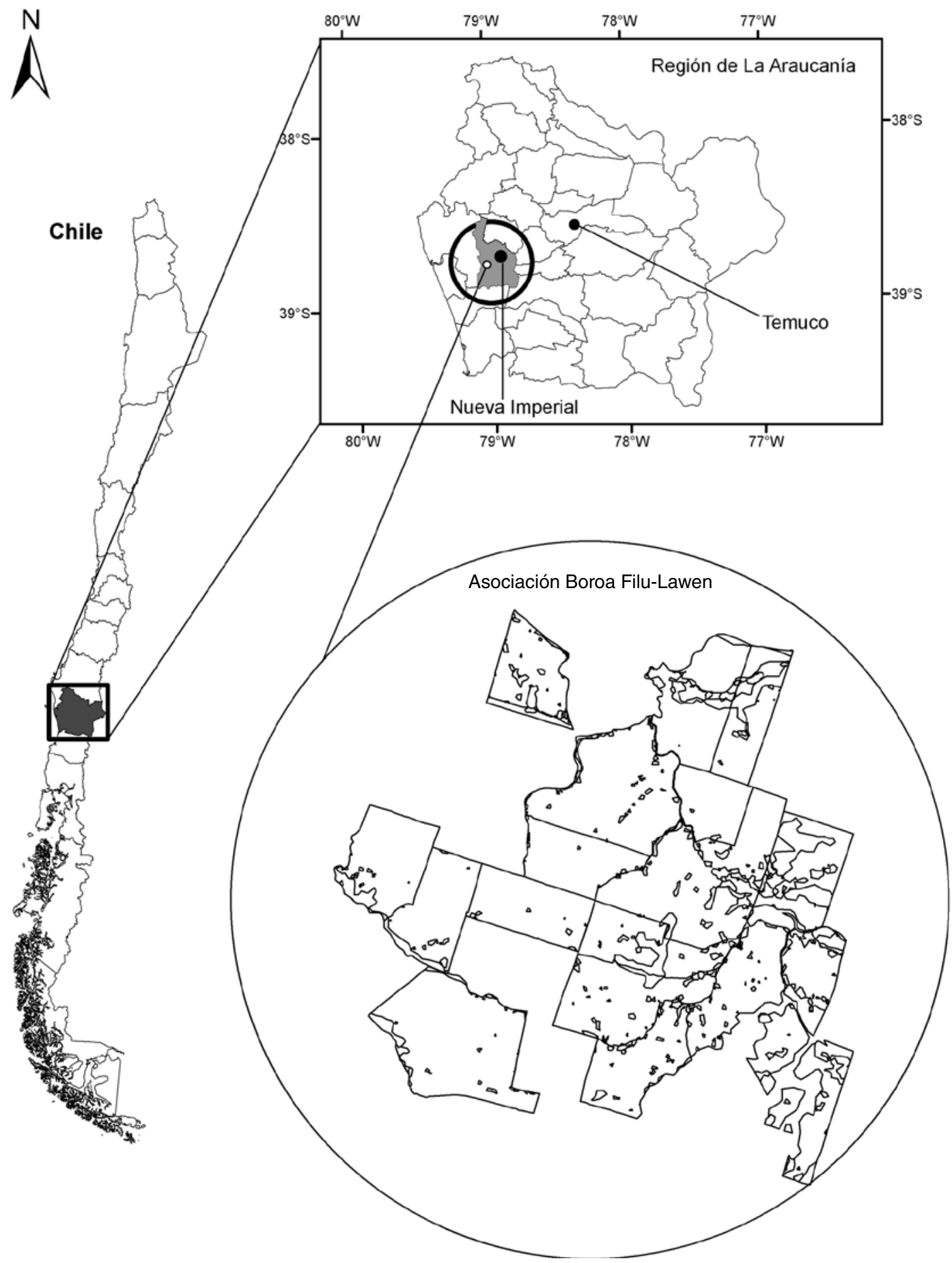

Figura 1. Mapa del territorio Boroa Filu-Lawen. Map of Boroa Filu-Lawen territory. 
depredaron ellos mismos los bosques, estableciendo pino y eucalipto para recibir dinero rápido. A pesar de estos problemas, en este territorio se ha ido dando un fuerte proceso de recuperación cultural, en que los jóvenes se hacen asesorar por kuifiche (ancianos) y kimunche o kimche (sabios), realizando ellos mismos este proceso de investigación para la recuperación cultural; con la Universidad establecieron cuatro reservas para proteger el bosque nativo y se plantean repoblar si fuera posible. Esta iniciativa generó apoyo en la comunidad, pero también críticas, tales como que los dirigentes han vuelto al pasado, no están con la modernidad, que la creencia en las machi y su visión de proteger el bosque nativo es retrógrada y va en contra del progreso necesario para el pueblo mapuche. Estas críticas no desalientan a los dirigentes, al contrario, reafirman la necesidad de conservar, revitalizar la cultura, respeto y reciprocidad con lo natural.

Este estudio explora a través del lenguaje -mapudungun- las categorías y significados vinculados a los diferentes espacios que congregan la vegetación (espacios ecológicos) del territorio Boroa-Filulawen según la percepción cultural de sus habitantes. Por lo tanto, los objetivos propuestos han sido: (1) identificar los espacios ecológicos significativos para la cultura mapuche en el territorio. En este contexto, importa dar cuenta de los elementos culturales que determinan la inclusión de todos los espacios identificados en la categoría "ecológico-cultural"; (2) caracterizar los espacios ecológico-culturales del territorio de acuerdo a criterios biofísicos que manejan los habitantes mapuche de la comunidad. Importa dar cuenta de los elementos topográficos, físicos $\mathrm{y}$ vegetacionales que definen al espacio como tal; y (3) describir los elementos culturales, físicos, ecológicos y vegetacionales asociados a cada espacio ecológico-cultural.

\section{Material y Método}

Se trata de un estudio cualitativo basado en la metodología de investigación acción participativa, la que consiste en un trabajo cooperativo entre investigadores de la universidad e investigadores locales mapuche. A través de esta investigación se conjugaron los intereses, motivaciones, y proyecciones de los investigadores y de la organización local para recuperar el conocimiento ancestral y las prácticas culturales de la comunidad.
El estudio implicó una primera fase de acuerdo con los dirigentes de la organización para coordinar una propuesta de investigación que tenga sentido para la comunidad, en este contexto se realizaron recorridos de campo, y discusión con líderes y ancianos mapuche. En una segunda fase denominada de diseño, se definieron e identificaron los participantes del estudio y la forma de recolección de datos. Los informantes fueron seleccionados por los dirigentes quienes reconocían en ellos la posesión de conocimiento ancestral mapuche. Con ellos se diseñaron sesiones de conversación grupal, entrevistas y recorridos de campo.

Se realizaron 10 sesiones grupales periódicas con 7 dirigentes mapuche ( 3 mujeres y 4 hombres entre 30 y 52 años), se entrevistó a 6 personas mapuche de la comunidad (4 mujeres y 2 hombres entre 96 y 45 años), las que fueron conducidas por un miembro de la organización en su propio idioma; y se entrevistó a un 'Machi' (principal agente curativo de la medicina mapuche); en total fueron entrevistadas 14 personas. Los recorridos periódicos a los espacios ecológicos permitieron realizar un registro visual de sus características y especies vegetales (Hoffmann 1997; Hoffmann et al. 2003). Con esto se reconocieron los lugares, se delimitaron los espacios significativos, y se obtuvo un registro de las especies vegetales de cada lugar. El recorrido permitió además identificar el protocolo mapuche de relación con la naturaleza.

La tercera fase, de análisis de los datos, consistió en la transcripción, organización y sistematización de las entrevistas realizadas a través de la técnica análisis de discurso (Rubio y Varas 2004). Una última fase consistió en establecer una serie de acciones tendientes a la protección de los espacios, especies vegetales y animales contenidas en ellos; y la distribución de cartillas en idioma "mapudungun" para la difusión de este conocimiento ancestral fuera y dentro de la comunidad.

\section{Resultados}

Los resultados del estudio se organizan de acuerdo a tres grandes áreas: identificación general de los espacios ecológicos significativos para la cultura mapuche en el territorio; caracterización de estos espacios de acuerdo a sus componentes biofísicos; y definición y descripción de las variables culturales, ecológicas y vegetacionales que caracterizan a cada uno de los espacios encontrados. 
Espacios ecológicos significativos culturales en el territorio Boroa-Filulawen

Se identificaron un total de 14 espacios ecológicos de significación cultural para el pueblo mapuche en el territorio de estudio, los cuales serán caracterizados en detalle más adelante. Los espacios más reconocidos y presentes en la comunidad fueron menoko, trayenko, lil, y fotrako, y los menos reconocidos fueron kilantu y riñintu (Tabla 1). Todos los espacios identificados fueron considerados culturalmente importantes debido a la presencia de ngen (dueño o administrador del espacio y de algunas especies vegetales) y newen (fuerza espiritual existente en todo el cosmos mapuche). Estos cuidan el lugar manteniendo las fuentes de agua, las especies vegetales, y confiriéndole además la fuerza y poder cultural al lugar. Los ngen son específicos de cada lugar y se presentan en diferentes formas, por ejemplo como mujer con cuerpo de pez, aves con cuerpo de culebras, trozos de cuero vivo, entre otros. Para evitar el enojo del ngen existe un protocolo cultural de ingreso y extracción o manipulación de alguno de sus componentes tales como plantas medicinales, alimentos o leña. Por ejemplo, pedir permiso a los ngen, hacer una rogativa llamada ngellipun, retribuir dejando un pago en monedas o algo vegetal, y comportarse respetuosamente con los seres que pueblan el lugar. Además existe una forma de recorrer los lugares y un horario específico de ingreso. En síntesis, es el ad Mapu del lugar, es decir, la normativa cultural de relación y uso de estos espacios lo que le confiere la sacralidad al lugar, normas que al no ser respetadas generarían daño o enfermedad a quienes las quebrantan. A modo de ejemplo uno de los participantes narró:

tenemos experiencia acá en Boroa; una persona se enfermó cuando fue a cortar leña al monte. Ella sin saber lo que había ahí, lo habría cortado y eso le produjo un tremendo susto. Una culebra la siguió y por la mitad del camino, para correr más fuerte se zafó el rebozo que llevaba y lo dejó tirao, después cuando fueron a ver y levantaron la ropa había una cabeza cortada de culebra y era con barba, según se decía se le pegó en la espalda de la señora, se enfermó y estuvo muy grave mucho tiempo, y falleció.
Otros elementos que confieren significación cultural a estos espacios son: utilización como sitios ceremoniales para rogativas relacionadas con el agua, lugar donde las machi reciben el don curativo; crecimiento de plantas con poder medicinal; y finalmente constituir espacios socioculturales de reproducción de la vida comunitaria, por ejemplo, lugar donde las mujeres lavan la lana en época de verano, conversan, refuerzan su identidad mapuche.

Considerando los aspectos mencionados, es posible plantear que todos los participantes compartieron la profunda convicción que la persona mapuche es un ser más de la naturaleza o cosmos, y en consecuencia debe respeto a cada uno de sus componentes en una relación de reciprocidad y cuidado.

\section{Elementos del medio físico que caracterizan los espacios ecológico-culturales}

La tipificación de los espacios ecológicoculturales está en directa relación con variables del medio físico que lo caracterizan; siendo muy relevantes: la existencia de agua en sus diversas formas, el tipo de vegetación existente y la topografía del lugar.

Nueve de los 14 espacios en el territorio (Tabla 1) fueron descritos a partir de su asociación con el agua. Esta tiene gran importancia en la cultura mapuche, lo cual se expresa en la incorporación del morfema ' $\boldsymbol{k o}$ ', en cinco de los espacios referidos, que en mapudungun significa agua. Entre estos: menoko (sitio pantanoso y presencia de vertiente), trayenko (agua que corre, cascada o chorrillo), fotrako (pantano o barrial), chayako (hoya con vertiente), y wiñoko (lugar donde un río o estero da una vuelta). Asimismo, se mencionaron el mallin (lugar inundado) y $\boldsymbol{l e w f u}$ (río), como espacios identificados a partir del agua.

Otra forma de describir estos espacios es de acuerdo a la especie vegetal predominante en el lugar, es así como se identifican a tres espacios: pitrantu (bosque de pitra), kilantu (bosque de kila), y riñintu o koliwal (bosque de koliwe).

La topografía del sector constituyó otra forma de identificar a los espacios; entre estos se mencionaron lil (quebrada barranco) y wingkul (monte o cerro pequeño).

Un caso especial es el denominado mawiza, ya que su característica esencial es la gran diversidad, abundancia y multiplicidad de especies nativas que 


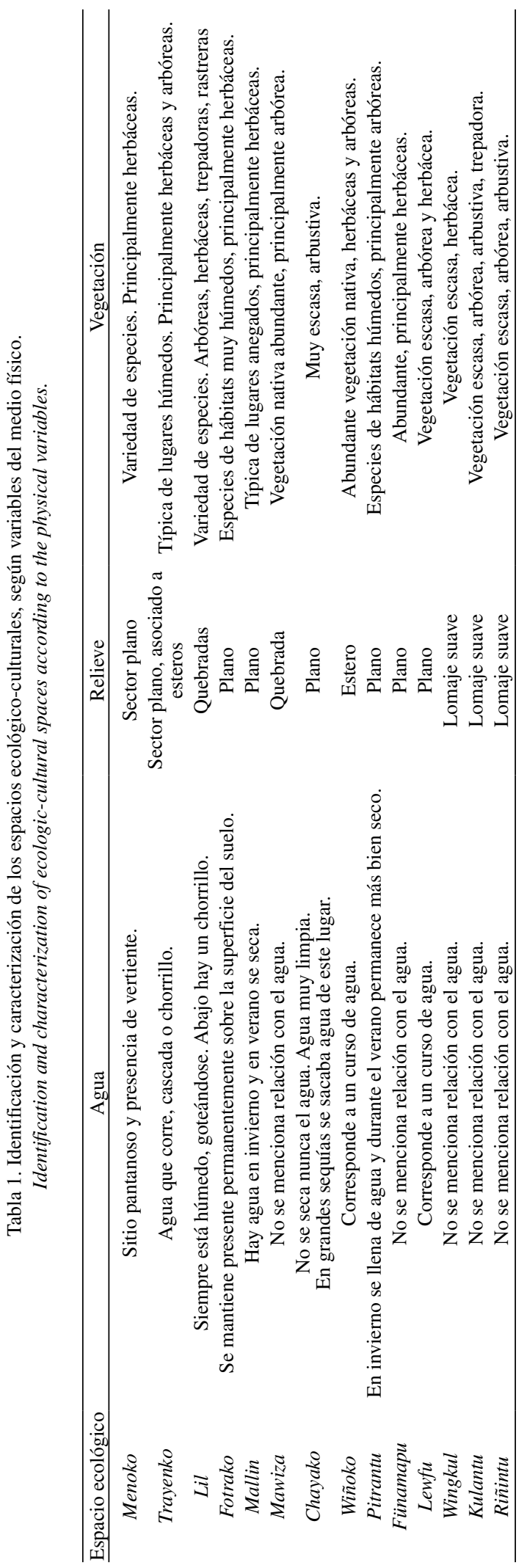

contiene. Es en resumen un espacio que puede contener a todos los anteriormente mencionados.

Finalmente, el espacio denominado funamapu, que literalmente significa tierra podrida, fue descrito por su fertilidad y vegetación medicinal; pero fue claramente diferenciado de los otros, por constituir un espacio generado a partir de la acción humana.

\section{Descripción cultural, ecológica y vegetacional de los espacios}

Los espacios identificados fueron a su vez descritos por su connotación cultural y la vegetación presente en el lugar. A continuación se describe cada uno de estos espacios.

\section{Menoko (Ojo de agua, vertiente o sitio pantano- so con abundante vegetación)}

Corresponde a un lugar sagrado y muy respetado por los habitantes del sector, ya que es el sitio preferencial del agente médico mapuche para la recolección de sus plantas medicinales; como expone uno de los entrevistados: "allí hay tierra viva, siempre hay agua, habitan muchas cosas para el bienestar de las personas, hay muchos remedios, mucha agua". Asimismo, se planteó que las enfermedades originadas por transgresiones culturales se expresan con mayor fuerza en estos espacios, y que su dueño espiritual se manifiesta al ser humano de distintas formas, en algunos casos aves, serpientes u otros animales. El menoko también puede ser el lugar en donde el agente médico mapuche experimenta el llamado divino a ejercer su rol sanador. Uno de los participantes señaló: "aquí se devuelve el rewue (símbolo de encuentro de la machi con sus espíritus en el momento del kimun [trance chamánico] confeccionado con un tronco de árbol sagrado) al menoko para que termine de morir". Todas estas condiciones hacen del menoko un lugar sagrado donde existen importantes fuerzas naturales y espirituales.

Desde un punto de vista ecológico, el menoko fue caracterizado por su alto grado de humedad, donde el agua en sus distintas formas siempre está presente. Algunos entrevistados lo definieron como 'un lugar de barro'; y otros como 'pantano o barrial'.

La vegetación existente se corresponde con una gran variedad de especies: herbáceas, arbustivas y helechos tales como: Solanum valdiviense L. (tomatillo), Samolus latifolius Duby (lechuguilla), Nertera 
granadensis (Mutis. Ex. L.F.) Druce (chaquirita del monte), Salvia officinalis (salvia), Solanum gayanum L. (natre), Myrceugenia correaefolia (Petrillo), Senecio fistulosus Poepp. Ex Less (hoja de paco), Blechnum chilense (Kaulf.) Mett. (costilla de vaca). Prácticamente toda la vegetación identificada tiene propiedades medicinales. Además en algunos menoko existen árboles como Drimys winteri J. R. et G. Forster (canelo), Myrceugenia exsucca (DC.) Berg (pitra), y Blepharocalyx cruckshanksii (H. et A.) Nied (temu).

\section{Trayenko (cascada-estero)}

Este espacio es un estero o chorrillo; una vertiente o pequeña cascada con piedras es conocido también como trayen o wichilko, el cual es menos frecuente de encontrar. Uno de los entrevistados lo describió como: "agua que emerge de la tierra', o lugar donde se juntan tres o más esteros: 'donde nace el agua y nunca se seca".

Este espacio tiene alta relevancia cultural, pues se utiliza para realizar rogativas en situaciones de inundación o sequía. Uno de los entrevistados menciona esto diciendo: "en el trayenko se hizo una Ceremonia-Rogativa-, luego de muchos días de lluvia apareció un caballo blanco, tomó agua, y el agua retrocedió". Existe otro tipo de trayenko en el que se realizan ceremonias para: "pedir agua cuando hay sequía: hay una fuerza que puede otorgar agua y cada comunidad sabe donde está su trayenko".

La vegetación más frecuente en este espacio son herbáceas, arbustivas y helechos como Equisetum bogotense H.B.K. (limpia plata), Cardamine nasturtioides Bertero (berro), Mentha polegium (poleo), Viola reichei Skottsb. (violeta), Chusquea quila Kunth (quila), Plantago major L. (llantén), costilla de vaca, y rastreras y trepadoras como Hydrocotyle ranunculoides L.f. (sombrerito de agua), Nertera granadensis (Mutis ex L.f.) Druce (chaquirita del monte), Cissus striata R. et P. (voqui naranjo) y árboreas como Aristotelia chilensis (Mol.) Stuntz (maqui), Peumus boldus Mol. (boldo), pitra. Cuando un trayenko ha sido intervenido la vegetación existente es escasa.

\section{Lil (quebrada o barranco)}

Este lugar fue descrito como una quebrada en donde 'se encuentran piedras, toscas, o piedras lajas', son lugares peligrosos y escarpados (pendiente de 60 a $90 \%$ aprox.). Pueden ubicarse en una montaña o cerca de un río y concluir en un chorrillo, estos lugares 'están goteando permanentemente, siempre húmedos'; y 'no pueden entrar los animales'. Se destaca el lugar más por el crecimiento de plantas medicinales que por sus características espirituales.

Con respecto a la vegetación, pueden ser herbáceas, arbustivas y helechos como Baccharis salicifolia (R. et P.) Pers. (refu), Ulex europaeus L. (pica pica), Discaria chacaye (G. Don) Tort (chacay), Schinus polygamus Cabr. (arrayán negro), Adiantum chilense Kaulf. (patitas negras), Gunnera chilensis (nalca), costilla de vaca y árboles como maqui, boldo y enredaderas rastreras o trepadoras como chaquirita del monte, Polypodium feuillei Bert (calahuala), Cissus striata R. et P. (voqui naranjo) y epifitas como Tristerix tetrandus (quintral del maqui).

\section{Chayako (lugar de rocas donde nace una vertiente)}

Lugar donde nace una vertiente y hay agua estacionada; un pozo con muchas rocas, una hoya o una piara, en donde el agua es muy pura y jamás se seca.

Este espacio tiene importancia sociocultural más que espiritual, pues era un lugar de encuentro para las mujeres, hacían fuego para cocinar, lavaban ropa, conversaban, tomaban mate, teñían lana y planificaban actividades de la comunidad. Este es uno de los espacios menos frecuente en el territorio.

Con respecto a la vegetación, pareciera ser más bien escasa, ya que la única especie arbustiva mencionada es Ribes magellanicum Poir. (zarzaparrilla).

\section{Mallin (vega)}

Se caracteriza por ser un lugar muy húmedo, donde los terrenos sufren anegamiento durante el invierno y sequía en el verano. Es una vega o un pajonal ubicado a orillas de cerros. El mallin se caracteriza por su importancia espiritual, porque está poblado de seres que cuidan o mantienen el agua. A diferencia de los anteriores, estos espacios pueden formarse también por acción del hombre; por ejemplo: 'es posible que el hombre cree mallines, si los terrenos se limpian, se mueve la tierra y se hacen hoyos'. En este territorio existe un mallin que antiguamente estaba cubierto por un bosque de temu, luego de la extracción de esta 
especie y del uso del terreno como pradera, el lugar comenzó a invadirse de junquillos, convirtiéndose finalmente en mallin. La época del año determina la importancia dada como lugar de crecimiento de plantas medicinales.

Desde el punto de vista vegetacional, el mallin estaría compuesto por herbáceas y arbustivas como Polygonum sanguinaria (sanguinaria), Mentha polegium L. (poleo), Myriophyllum aquaticum (Vell.) Verdc. (pasto pinito), Coriaria ruscifolia L. (mata ratones), Juncus procerus E. Meyer (junquillo), Sagittaria montevidensis Cham. et Schecht. (flecha de agua), chacay, refu, arrayán negro, y rastreras como sombrerito de agua, además de arbóreas como Salix sp. (sauce).

\section{Pitrantu (bosque de pitra)}

Es un bosque de pitra de individuos jóvenes y bajo crecimiento, de ambiente húmedo en invierno y seco en época estival; es descrito como bosque pantanoso que se desarrolla a orillas de cursos de agua y que permanece anegado gran parte del periodo invernal. Este espacio posee también un dueño espiritual que cuida el bosque. Tiene un número significativo de especies medicinales, entre ellas el canelo, planta sagrada en la cosmovisión mapuche por su utilización en ceremonias rituales de la comunidad.

Con respecto a la vegetación crecen especies presentes de ambientes húmedos, trepadoras como calahuala, epífita como Notanthera heterophylla (R. et P.) Barl. et Wiens. (quintral del boldo), y arbóreas como maqui, canelo, Blepharocalyx cruckshanksii (temu) y pitra.

\section{Mawiza (montaña-bosque)}

Es un monte de abundante vegetación nativa, densa, habitualmente ubicado en los cerros. Este contiene la mayor diversidad de especies vegetales y animales, por lo general es de mayor extensión y puede contener todos los espacios mencionados anteriormente. Uno de los entrevistados expresa esta diversidad diciendo: "en el mawiza se encuentran todas las cosas, hospedajes de aves, peucos, chingues, buitres, zorros, horcones y lechuzas". Por su gran diversidad vegetacional provee a las comunidades de plantas de uso medicinal y de teñido, forraje para los animales, leña y alimento. Desde el punto de vista cultural se caracteriza por la presencia de múltiples fuerzas espirituales frente a las cuales los seres humanos deben tener un comportamiento respetuoso, cumpliendo una serie de normas. Como se mencionó en secciones anteriores, en el mawiza las personas deben hacer una rogativa y evitar comer, tomar leche, dormir, reírse o jugar en el sector; una transgresión muy grave para los habitantes de Boroa es maltratar los animales que allí habitan.

Con respecto a la vegetación existen todo tipo de árboles, lo que nos indica que es un lugar de gran diversidad y abundancia. Las especies arbustivas son Arrayán negro, Buddleja globosa Hope (matico), Chusquea coleu Desv. (colihue), quila, y zarzaparrilla, árboles como Maytenus boaria Mol. (maitén), boldo, Laurelia sempervirens ( $\mathrm{R}$. et P.) Tul. (laurel), Persea lingue (R. et P.) Nees ex Kopp (lingue), Nothofagus obliqua (Mirb.) Oersted (roble), y canelo.

\section{Lewfu (río)}

Es un curso de agua de un tamaño considerable, mayor que un estero o chorrillo. Desde el punto de vista cultural existen seres espirituales que habitan en el agua, los cuales pueden causar la muerte por inmersión a quienes se bañan en horarios inapropiados, o bien cuando se transgreden normas culturales de uso.

La vegetación corresponde a formaciones de ribera tales como sauce, maqui, arrayán negro, maitén, Rosmarinus officinalis L. (romero), Ulex europaeus L. (pica-pica) y Cirsium vulgare (Savi) Ten (cardo).

\section{Wiñoko (río que cambia de curso)}

Es un lugar en donde un estero o río desvía su curso, es un sitio donde crecen plantas medicinales de gran importancia para la medicina mapuche. Es un espacio formado por acción de los años, se puede dar arriba entre ramas de los árboles, y en donde se acumula agua de color café. En este lugar crecen pequeñas plantas de gran poder medicinal. $\mathrm{Al}$ igual que en los espacios anteriores existen en él fuerzas espirituales.

Desde el punto de vista vegetacional hay gran cantidad de plantas medicinales, entre ellas herbáceas como berro, lechuguilla, violeta; helechos como costilla de vaca; rastreras como chaquirita del monte; trepadoras como voqui naranjo; y arbóreas como laurel, maqui. 


\section{Kulantu (bosque de kila o quila)}

Corresponde a un 'bosque de quila', y se describe como un lugar de gran cantidad de estas especies creciendo juntas, con amplia cobertura de tierra, y donde prácticamente nada más puede crecer. Culturalmente se indica la presencia de nidos de serpientes como dueños espirituales que cuidan este espacio. No se menciona uso medicinal de la quila, sin embargo, la floración de esta especie es indicador de desgracias para la comunidad mapuche.

Con respecto a la vegetación esta resulta ser escasa, encontrándose una arbustiva, quila, una trepadora Lapageria rosea Ruiz et Pavón (copihue) y una arbórea, maqui.

\section{Riñintu (bosque de colihue)}

Se describe como un grupo extenso de colihues creciendo juntos, generalmente en formaciones aisladas. Culturalmente este espacio tiene dueño espiritual al igual que todos los anteriores. Aunque no se indicaron usos medicinales para el colihue, su importancia es alimenticia ya que antiguamente sus brotes, previamente cocidos en agua, eran preparados en ensaladas. Además es considerado como una planta protectora para la regeneración de otras especies.

En este espacio crecen varias otras especies de uso medicinal, alimenticio, y arbustivas como colihue, zarzaparrilla y arrayán negro, arbóreas como maqui, Sophora microphylla Ait. (pelú).

\section{Fotrako (pantano)}

Es un lugar con barro, pantanoso, donde siempre existe agua superficial, permitiendo que algunos sectores no se sequen nunca. Culturalmente, igual que los otros espacios, presenta un dueño espiritual y seres que se representan en distintas formas.

En cuanto a la vegetación estaría conformada principalmente por especies herbáceas tales como pasto pinito, berro, hoja de paco, junquillo, Rumex patientia (romaza), ratonera, Marrubium vulgare L (toronjil cuyano), helecho costilla de vaca, una rastrera: sombrerito de agua y una arbórea: canelo. Fünamapu (tierra fértil, tierra podrida).

A diferencia de los anteriores este espacio es creado por la acción del hombre. Son tierras cercanas a la vivienda, que desprovistas de su vegetación original han sido destinadas previamente para corrales de animales, y en donde al erradicarlos crece una serie de especies herbáceas, muchas de éstas presentes en huertas familiares. Posterior a la erradicación de los animales, este espacio es colonizado con una diversidad de especies de uso medicinal. Culturalmente presenta un dueño espiritual pero no se considera lugar sagrado.

La vegetación presente sería principalmente herbácea como Urtica urens L. (ortiga blanca), Urtica dioca L. (ortiga negra), Chenopodium quinoa Willd (quinoa), cardo, poleo, Matricadia chamomilla (manzanilla), Conium maculata (cicuta), Chenopodium ambrosoides L. (paico), Datura stramonium L. (chamico), Taraxacum officinale G. Webwe ex Wigg. (diente de león), sanguinaria, toronjil cuyano, Eleocharis pachycarpa Desv. (pasto de caballo), Plantago lanceolada L. (siete venas).

\section{Wingkul (cerro o loma)}

La descripción de este espacio presentó opiniones diversas respecto de sus características biofísicas. Algunos lo describen como un cerro o una pequeña loma, y otros como espacios planos entre cerros o en los cerros. También se describen como cerros con vegetación nativa, en donde algunos sectores están libres de vegetación, por lo cual se utilizan para cultivo. Culturalmente este espacio tendría una connotación religiosa importante, pues en él se realizan ceremonias mapuche de sanación.

Las especies asociadas al wingkul son herbáceas como sanguinaria, tomatillo y la arbórea laurel.

\section{Discusión}

Los resultados evidencian que la concepción mapuche de los ecosistemas es similar a otros pueblos indígenas en Latinoamérica (Rist 2006). Esta implica una cosmovisión que concibe al ser indígena parte constitutiva de la naturaleza, como un elemento más en la cadena ecológica. Por lo tanto, se sugiere que el ser mapuche es parte de y está sometido a distintas fuerzas espirituales que componen su mundo natural. Desde esta perspectiva los espacios ecológicos identificados son descritos no sólo en tanto a sus características biofísicas, sino, además, por las significaciones culturales, sociales, o ceremoniales. Por esta razón los espacios descritos constituyen una unidad ecológica y cultural para los habitantes del sector. 
Lo cultural está dado, en cada uno de los espacios, por la presencia de dueños y fuerzas espirituales quienes cuidan sus animales, plantas y fuentes de agua. Las fuerzas o energías espirituales que gobiernan en el espacio otorgan ciertos poderes a los elementos contenidos en él; es así como plantas, animales, agua o barro adquieren significación para la cultura mapuche. En consecuencia, si los espacios tienen dueños y seres que los habitan, las personas deben seguir un conjunto de normas culturales que regulan la relación con éstos; y su transgresión puede derivar en daño, enfermedad o incluso la muerte. La presencia de estos seres demuestra que para la persona mapuche, la naturaleza no es un agregado de recursos bajo potestad del ser humano; al contrario, sociedad y naturaleza portan espíritu y conforman un todo relacional. En consecuencia, en un mismo espacio conviven fuerzas visibles o materiales, con otras más sutiles y de apariencia diversa.

Comparado con otros autores, se coincide con Pérez (2004) quien menciona mallin, menoko, mawiza y wingkul. Por otra parte, se coincide con Durán et al. (1998) en mallin, wingkul, lil y mawiza. La diferencia observada entre estos estudios puede fundamentarse no sólo a las condiciones objetivas físicas del espacio, sino también a diferentes denominaciones locales y a diferencias en el manejo del conocimiento cultural y memoria colectiva.

Gran parte de las especies mencionadas son de uso medicinal, combinado con uso alimenticio, forrajero, combustible, maderero o teñido.

La identificación y descripción de los espacios desde la perspectiva cultural demuestran una diferencia significativa con las de la sociedad científica u occidental. Hay, por ejemplo, mayor diversidad en las denominaciones culturales, mayor descripción de los detalles del sitio y mayor riqueza o complejidad en las descripciones. Ello demostraría una diferencia en la cosmovisión cultural del entorno, que como se ha expresado en la población indígena integra lo natural y espiritual.

La recuperación del conocimiento tradicional contribuiría, por un lado, a fortalecer la memoria colectiva de significado para la vida personal, espiritual, familiar y del bienestar de la comunidad. También debiera mejorar los diseños de manejo de recursos naturales incorporando como eje del modelo el resguardo y respeto por los ecosistemas y culturas tradicionales locales.

Para los participantes de este estudio, la comprensión del ser mapuche requiere un proceso de reforzamiento dentro y fuera de la propia cultura, con el propósito de recuperar y fortalecer el conocimiento ancestral, lo que contribuiría en el mediano y largo plazo no sólo a proteger o rescatar los espacios perdidos, sino también a valorizar la identidad mapuche en el contexto sociopolítico nacional.

Agradecimientos: Esta investigación ha sido posible gracias al apoyo obtenido del proyecto DIDUFRO No 120302 y DIUFRO DI08-0064 Universidad de La Frontera. Temuco-Chile.

A la Coordinadora de Salud Intercultural Boroa-Filulawen, a sus dirigentes Antonio Huircan Pichicon, Abelino Pichicona Guimay, Carmen Pichicona Guimay, Braulio Cheuquepal Baeza, Gladys Cheuquepal Baeza; a la comunidad de Boroa, al Machi Víctor Canuillan, y a los evaluadores anónimos de este artículo.

\section{Referencias Citadas}

Altieri, M. 2003. The sociocultural and food security impacts of genetic pollution via transgenic crops of traditional varieties in Latin American Centers of Peasant Agriculture. Bulletin of Science, Technology \& Society 23:1-10.

Antivil, A. 2004 Impacto de los Programas de Desarrollo en Comunidades Mapuche: Región de La Araucanía. Tesis para optar al título de Ingeniero Forestal. Facultad de Ciencias Agropecuarias y Forestales, Universidad Católica de Temuco, Temuco.

Bragg, K., E. Hauenstein y M. Latsague 1986. Transecto etnobotánico del sector mapuche. Cultura, Hombre, Sociedad 3:57-80.

CIREN 2010. Determinación de la Erosión de los Suelos de Chile Región de La Araucanía. Servicio Agrícola y Ganadero, Instituto de Desarrollo Agropecuario, Corporación Nacional Forestal, Gobierno de Chile, Santiago.
Citarella, L., A. Conejeros, B. Espinosa, I. Jelves, A. Oyarce y A. Vidal (eds.) 2000. Medicinas y Culturas en La Araucanía. Editorial Sudamericana, Santiago.

COMITÉ DE SALUD INTERCULTURAL BOROA FILULAWEN 2002. Documento creación del centro de salud integral para el territorio Mapuche Boroa-Filulawen Comuna Nueva Imperial. IX Región de La Araucanía.

CONAF Y CONAMA 1999. Catastro y Evaluación de Recursos Vegetacionales Nativos de Chile. Informe Regional IX Región. Universidad Austral de Chile, Pontificia Universidad Católica de Chile y Universidad Católica de Temuco.

Durán, T., J. Quidel, E. Hauenstein, L. Huaiquimil, Z. Hinostroza y E. Pichun 1998. Conocimientos y vivencias de dos familias wenteche sobre medicina. Cultura, Hombre, Sociedad 9:83-91. 
Gajardo, R. 1994. La Vegetación Natural de Chile: Clasificación y Distribución Geográfica. Editorial Universitaria, Santiago, Chile.

Grebe, M.E., S. Pacheco y J. Segura 1972. Cosmovisión mapuche. Cuadernos de la Realidad Nacional 14:46-73.

Gumucio, J.C. 1989. Los vegetales como el reflejo del saber de un pueblo. El modelo mapuche. Nütram 4:25-36.

Gusinde, M. 1936. Plantas medicinales que los indios araucanos recomiendan. Anthropos 556-571.

- _ - 1938. Medicina e Higiene entre los Araucanos. Tomos I-II. Publicaciones del Museo de Etnología y Antropología de Chile, Santiago.

Hill, R. y C. Sendashonga 2006. Conservation biology, genetically modified organisms, and the biosafety protocol Conservation Biology 20:1620-1625.

Hoffmann, A. 1997. Flora Silvestre de Chile. Zona Araucana. Ediciones Fundación Claudio Gay, Santiago.

Hoffmann, A., C. Farga, J. Lastra y J. Veghazi 2003. Plantas Medicinales de Uso Común en Chile. Ediciones Fundación Claudio Gay, Santiago.

INE 2002. Etnia 30 abril 2008 http://www.ine.cl/cd2002/etnia.pdf.

Kristensen, M. y H. Balslev 2003. Perceptions, use and availability of woody plants among the Gourounsi in Burkina Faso. Biodiversity and Conservation 12:1715-1739.

Lara, A., I. Araya, J. Cabello, M. Fierro y A. Cavieres 1987. Evaluación de la destrucción y disponibilidad de los recursos forestales nativos en la VII y VIII regiones. CODEFF, WWF, IUCN, Santiago.

La Torre, M. y G. Islebe 2003. Traditional ecological knowledge and use of vegetation in southeastern Mexico: A case study from Solferino, Quintana Roo. Biodiversity and Conservation 12:2455-2476.

Mackinnon, P. y S. McFall 2000. Pueblo Mapuche, Expansión Forestal y Poder Local. Programa Mapu-Territorialidad. Municipio y Participación (o exclusión) Mapuche. Documento inédito Centro de Documentación del Instituto de Estudios Indígenas, Universidad de La Frontera, Temuco.

Marileo, A. 1995. Modernización o Sabiduría Mapuche. Ed. San Pablo, Temuco.

- - _ 2000. Mundo Mapuche. En Medicinas y Culturas en La Araucanía, editado por L. Citarella, A. Conejeros, B. Espinosa, I. Jelves, A. Oyarce y A. Vidal, pp. 58-63. Editorial Sudamericana, $2^{\mathrm{a}}$ ed., Santiago.
_ _ _ 2001. Mundo Mapuche. Memoria del Primer Seminario Taller: Cosmovisión Indígena y Biodiversidad en América Latina. Comunidad Chorojo, Cochabamba-Bolivia.

Marvier, M.A. 2002. Improving risk assessment safety of transgenic crops. Ecological Applications 12:1119-1124.

MUNICIPALIDAD DE NUEVA IMPERIAL 2004. Diagnóstico Técnico Plan de Desarrollo Comunal (2002-2005). Comuna Nueva Imperial-Secretaría de Planificación. http://www.nuevaimperial.cl/plan_desa.htm (7 marzo 2009).

Ñanculef, J. 1989. El concepto territorial en el pueblo mapuche. Nütram 4:5-9.

ODEPA 2000. Clasificación de las explotaciones agrícolas del VI Censo Nacional Agropecuario según tipo de productor y localización geográfica. Documento de Trabajo No 5 , Santiago.

Pérez, I. 2004. Ecosistemas Mapuche: Diálogo Intercultural para la Restauración Ambiental en la Región de La Araucanía. Tesis para optar al grado de Magíster en Educación, Mención Educación, Facultad de Educación, Universidad de La Frontera, Temuco.

- _ _ 2005. Marco conceptual para la restauración de ecosistemas en territorio Mapuche: Compartimos un pedacito del mundo en estos confines del Sur. Serie Publicaciones 4:1-29.

Rist, S. 2006. Diálogo Intra e Intercientífico entre Comunidades Ontológicas: Caminos para Recuperar las Dimensiones Espirituales de las Ciencias Naturales y Sociales. Agruco Compas. Plural Editores, La Paz.

Rubio, M. y J. Varas 2004. El Análisis de la Realidad de la Intervención Social. Métodos y Técnicas de Investigación. Ediciones CCS, Madrid.

Santibáñez, F. y M. Uribe 1993. Atlas Agro climático de Chile: Regiones Sexta, Séptima, Octava y Novena. Universidad de Chile, Facultad de Ciencias Agrarias y Forestales. Laboratorio de Agro-climatología. Departamento de Ingeniería y Suelos, Santiago.

Toledo, V. 1990. La perspectiva etnoecológica: cinco reflexiones acerca de la ciencia campesina sobre la naturaleza con especial referencia a México. Ciencia, Número Especial 4:22-29.

Villagrán, C., M. Romo y V. Castro 2003. Etnobotánica del sur de los Andes de la Primera Región de Chile: un enlace entre las culturas altiplánicas y las de quebradas altas del Loa superior. Chungara Revista de Antropología Chilena 35:73-124. 
